\title{
Phytotherapie im peripartalen Zeitraum bei Hund und Katze (Praxiserfahrungen)
}

\author{
Cäcilia Brendieck-Worm
}

Arbeitskreis Phytotherapie der Gesellschaft für Ganzheitliche Tiermedizin, Niederkirchen, Deutschland

\section{Arzneipflanzen zur Unterstützung der Mutter}

Ein präventiver und therapeutischer Einsatz von Arzneimitteln beim graviden und laktierenden Tier muss grundsätzlich die Welpen berücksichtigen. Dies gilt sowohl für Arzneimittel synthetischer als auch pflanzlicher Herkunft. Viele Therapeutika können die Plazentar- und die BlutMilch-Schranke überwinden. Bei geringer therapeutischer Breite gefährdet möglicherweise die Medikation der Mutter die Welpen. Im Folgenden wird auf gut verträgliche pflanzliche Zubereitungen für Mutter und Welpen im peripartalen Zeitraum eingegangen.

\section{Mütterliche Mikroflora optimieren und schützen}

Pflanzliche Zubereitungen können die Gesundheit des Muttertiers ante partum optimieren, was letztlich dem Welpen zugutekommt und diesem den Start in das eigenständige Leben erleichtert.

Besondere Aufmerksamkeit gilt der mütterlichen Mikroflora, denn eine gesunde Mundhöhlen-, Darm-, Vaginalund Hautflora der Mutter garantiert eine optimale Besiedelung des Neugeborenen und legt damit den Grundstein für die Entwicklung eines kompetenten Immunsystems beim Jungtier. Eine gestörte maternale Mikroflora, z.B. die Besiedelung von Parodontaltaschen mit pathogenen Erregern oder eine vaginale Candidose als Folge einer gestörten Darmflora, verursachen eine Fehlbesiedelung beim Säugling. Auch der unkritische Umgang mit Antiseptika und Antibiotika bei der Geburtshilfe und Gesäugepflege führen beim Säugling zur Dysbiose. Die Geburt durch Sectio caesarea bedeutet gar für das Neugeborene die Erstbesiedelung mit Praxis-/Klinikkeimen, was eine nicht zu vernachlässigende Gefahr für nosokomiale Infektionen beim Welpen darstellt.

Die Kontrolle und gegebenenfalls Sanierung von Mundhöhle, Darm und Geburtsweg sowie die Optimierung von Verdauung und Stoffwechsel sind deshalb die Voraussetzung für gesunde Welpen.

\section{KARGER}

Fax +497614520714

Information@Karger.com

www.karger.com
Phytotherapie bei gestörter Mundflora

Während der Gravidität kann die Lockerung des Bindegewebes das Eindringen pathogener Erreger begünstigen. Dies zeigt sich häufig im Bereich der Mundhöhle. Ante partum auftretende Zahnfleischentzündungen, Zahnbeläge und Biofilmbildung können bei der Hündin durch Propolislösungen [1], Sprays und Gelzubereitungen mit ätherischem Lemongrasöl [2] und anderen ätherischen Ölen therapiert werden.

\section{Phytotherapie bei gestörter Vaginalflora}

Dysbalancen der Vaginalflora können bereits zu Problemen bei der Bedeckung der Tiere führen. Bei Vaginitiden hat sich Lavendelöl bewährt. Gute Schleimhautverträglichkeit, breites antimikrobielles Spektrum sowie antiphlogistische, heilungsfördernde, juckreiz- und schmerzstillende Wirkungen [3] machen es zu einem geeigneten Therapeutikum auch während der Gravidität (Vaginalovula, -cremes). Geburtsverletzungen von Vulva und Vagina lassen sich ebenfalls mit Lavendelölzubereitungen therapieren. Vorsicht ist bei der Katze geboten.

Candida-Vaginitis und Infektionen mit Trichomonas vaginalis können mit Teebaumöl (Suppositorien) erfolgreich behandelt werden [4]. Der lokale Einsatz von Teebaumöl ist eine Alternative zu Nitroimidazolen, die im Verdacht stehen, mutagen und kanzerogen zu sein. Für Katzen ist Teebaumöl toxisch und darf grundsätzlich nicht eingesetzt werden.

Bei Geburtsverletzungen im Bereich von Vulva und Vagina bewähren sich Spülungen mit Zubereitungen aus Kamillentinktur.

Phytotherapie bei gestörter Darmflora und dyspeptischen Beschwerden

Insbesondere bei Fehlbesiedelungen im Darm treten in der Hochträchtigkeit nicht selten dyspeptische Beschwerden auf (Meteorismus, Flatulenz, Appetitlosigkeit). Hier bewähren sich bei der Hündin Karminativa wie Kümmel und Fenchel sowie Bitterstoffdrogen. Diese wirken sekretionsstei- 
gernd auf Speicheldrüsen, Magen, Galle und Pankreas, motilitätsfördernd und spasmolytisch auf Magen, Gallengänge und Darm, leberschützend und -regenerierend sowie appetitanregend. Für Katzen sind Bitterstoffe nur in potenzierter Form geeignet (D1/D2). Zu den bewährten Bitterstoffdrogen zählt der Löwenzahn. Er wirkt zudem diuretisch und reduziert die Ödem-Neigung. Die lipid- und triglyzeridsenkende und blutzuckerregulierende Artischocke empfiehlt sich insbesondere bei der adipösen Hündin. Obstipation kann bei Hündin und Kätzin durch pflanzliche Füll- und Quellstoffe wie Leinsamen und Flohsamen(-schalen) behoben werden. Allerdings ist hier eine ausreichende Flüssigkeitsaufnahme eine wichtige Voraussetzung. Pflanzliche Drastika sind während der Gravidität aufgrund ihrer Wirkung auf den Uterus kontraindiziert.

Zur Unterstützung der Darmflora empfehlen sich Präbiotika wie z.B. Apfelpektin oder Inulin-haltige Pflanzenzubereitungen (Zichorienwurzel).

\section{Pflanzliche Unterstützung im Geburtsverlauf}

Kaffee als pflanzliches Analeptikum hilft der durch einen langen Geburtsprozess geschwächten Mutter. Seine Methylxanthine werden rasch resorbiert und in alle Gewebe verteilt und passieren die Blut-Hirn-, die Blut-Plazentarsowie die Blut-Milch-Schranke. Sie wirken zentral stimulierend und positiv inotrop; der Blutdruck wird kurzfristig leicht erhöht. Die Nierendurchblutung steigt. Als Wechselwirkungen sind Wirkungsverstärkung bei Digitalispräparaten und $\beta$-Sympathomimetika (z.B. Tokolytika) zu beachten [5]. Einsetzbar ist sowohl der in jedem Haushalt verfügbare Kaffee als Infus mit Milch und Zucker als auch das als Arzneimittel zugelassene Decoctum Coffea praeparata. Bereits nach wenigen Minuten verbessert sich das Allgemeinbefinden. Die Atmung normalisiert sich. Das Interesse an den Welpen erwacht und das Gesäuge füllt sich. Nach der Kaffeegabe geborene Welpen sind vitaler.

\section{Arzneipflanzen für die aggressive/hyperaktive und ängstliche Mutter}

Aggressionen der Mutter werden für das Neugeborene schnell lebensbedrohlich, ebenso eine nervöse/ängstliche Mutter. Diese neigt zu übertriebener Neugeborenenpflege: Starkes Belecken kann zu Schäden an Nabel und Bauchdecke führen [6].

Mütterliche Unruhe und Hektik als Reaktion auf Lautäusserungen von Welpen oder Ruhestörung durch (fremde) Personen können zu plötzlichem Herumwerfen und Totdrücken/-treten von Welpen führen. Auch rastloses Herumtragen der Jungen, wie es bei ungeeigneter Wurfkiste und Unruhe im Umfeld beobachtet werden kann, schadet den Welpen. Bewährt haben sich bei psychisch bedingten Verhaltensstörungen der Mutter während und nach der Geburt insbesondere die ätherischen Öle von Kamille, Lavendel, Melisse und Muskatellersalbei [7]. Ihre Inhalation über die Duftlampe wirkt angstlösend sowie stressmindernd und führt zur Beruhigung der Mutter. Diese Therapie ist im Gegensatz zur ansonsten üblichen Gabe von Acepromazin für Mutter und Säugling frei von unerwünschten Nebenwirkungen.

Phytotherapeutische Einflussnahme auf die Zusammensetzung der Muttermilch

Kolostrum enthält viele fettlösliche Vitamine. Besonders der Gehalt an Vitamin A und E ist dabei stark von der Fütterung der Mutter ante partum abhängig. Da nur geringe Mengen fettlöslicher Vitamine die Plazentarschranke überwinden können und zudem die fetale Speicherkapazität gering ist, ist eine ausreichende Kolostrum-Aufnahme essenziell [6]. Mangel an Vitamin A und Provitamin A ( $\beta$-Carotin) kann zur Epithelschädigung von Auge und Schleimhaut sowie zu Wachstumsstörungen, Immunschwäche, oxidativem Stress und Anämie führen. Vitamin-E-Mangel verursacht Muskelschwäche, Hämolyse, oxidativen Stress und Resorptionsstörungen. Während die carnivore Katze ihren Vitamin-A-Bedarf über tierische Nahrung wie Leber, Eidotter usw. decken muss [8], können Hunde vom $\beta$-Carotingehalt von Pflanzen wie Karotte, Tomate, Kresse, Hagebutte, Paprika, Mais und Sanddorn profitieren. Vor allem Weizenkeim-, Maiskeim-, Sonnenblumen-, Sanddorn-, Oliven- und Palmkernöl sowie Mandeln und Haselnüsse enthaltend Vitamin E.

Weitere wichtige Vitamine werden durch die gesunde Darmflora der Mutter synthetisiert, so z.B. Phyllochinon. In den ersten Lebenswochen ist die Zufuhr von Phyllochinon über die Milch essenziell, da die enterale Eigensynthese noch zu gering ist, um eine funktionsfähige Blutgerinnung zu gewährleisten [6]. Auch Cobalamin (antianämische Wirkung) wird ausschliesslich von Darm-Mikroorganismen gebildet.

\section{Arzneipflanzen zur Steigerung der Milchmenge}

Zur Steigerung der Milchmenge und Regulation des Milchflusses beim Hund eignen sich Teezubereitungen aus Fenchel-, Anis- und Kümmelfrüchten und Brennnesselblättern zu gleichen Teilen. Die karminative Wirkung des Kümmels geht dabei in die Milch über und bewahrt den Säugling vor Blähungen.

Ausserdem wirken Aufgüsse von Bockshornkleesamen (Kaltansatz) milchflussfördernd und anregend auf das Wachstum der Milchdrüse [9]. Diese phytotherapeutischen Optionen sind der in der konventionellen Medizin empfohlenen Anwendung von Metoclopramid mit ihren möglicherweise schwerwiegenden Nebenwirkungen für Mutter und Säugling weit überlegen.

\section{Gesäugegesundheit}

Die Pflege des insbesondere zum Ende der Laktation stark strapazierten Gesäuges geschieht am besten durch auch für Welpen geeignete milde pflanzliche Öle. Reizmildernd wirken zudem mehrmals täglich aufgelegte Fenchelteekompressen oder Waschungen mit Fenchel- oder Ringelblumentee. 
Bei stark zerkratztem Gesäuge empfiehlt sich Ringelblumenauszugsöl. Verlässt eine ängstliche und unruhige Mutter bei schmerzhaftem Gesäuge ihre Jungen, kann die Aromatherapie hilfreich sein.

\section{Arzneipflanzen als Starthilfe für Welpen}

Die wichtigsten pharmakokinetischen Parameter (Halbwertszeit, Kumulation, Verteilungsvolumen, Plasmaproteinbindung, Bioverfügbarkeit) entsprechen beim Neugeborenen und Säugling nicht denen von erwachsenen Tieren. Im Vergleich zum Adulten verfügen sie über weniger Albumine, was die Ödem-Neigung insbesondere bei fehlendem Kolostrum erklärt. Die Blut-Hirn-Schranke ist noch ineffektiv, der «first pass effect» meist geringer, das Blutvolumen im Verhältnis zur Körpermasse grösser und der Anteil extrazellulärer Flüssigkeit höher, was sich in höherer Harnmenge und niedrigerer Harnmolarität zeigt. Die Nierenfiltrationsrate liegt deutlich unter der erwachsener Tiere. Plasmamolarität und -volumen sowie Blut-pH schwanken noch erheblich $[5,6]$.

Die Pharmakotherapie von Neugeborenen und Säuglingen birgt deshalb Risiken. Phytotherapeutika erweisen sich erfahrungsgemäss als besser verträglich und nebenwirkungsärmer als Synthetika. Dies gilt allerdings aufgrund der Nahrungsspezialisierung auf Fleisch und damit fehlender evolutionärer Anpassung an Pflanzen nur mit grossen Einschränkungen für Feliden (Glukuronidierungsschwäche).

\section{Atemstimulation bei lebensschwachen Welpen}

Asphyxie durch Sauerstoffmangel während der Geburt durch Nabelkompression, vorzeitige Plazenta-Ablösung oder durch Narkotika (Sectio caesarea) kann durch pflanzliche Atemstimulanzien positiv beeinflusst werden. Bei den Narkotika geschieht dies begleitend zur Injektion von Antagonisten.

Bewährt haben sich bei Hunde- und Katzenwelpen Ätherisch-Öl-haltige Sprays zum Aufsprühen auf die Nase, die unter anderem mukolytisch, sekretolytisch, bronchospasmolytisch, psychoaktivierend und tonisierend wirkende ätherische Öle aus Fenchel, Nelken, Melisse usw. enthalten. Als orales Atemanaleptikum wirkt der Kaffee, hier vorzugsweise das Arzneimittel Coffea praeparata oral.

\section{Infektabwehr des Neugeborenen}

Der Übergang vom weitgehend sterilen Uterus in die keimreiche Aussenwelt ist ohne Kolostrum, das speziell auf das Keimspektrum der Umgebung ausgerichtete Antikörper bereithält, für Hunde- und Katzenwelpen lebensgefährlich. Die körpereigenen Möglichkeiten des Neugeborenen sind noch zu langsam und zu unvollständig, um Infektionen standzuhalten. Deshalb erkranken Neugeborene, die kein Kolostrum erhalten haben, erheblich schneller an Coliseptikämien, Diarrhöen und Pneumonien. Offenbar spielt das Kolostrum bei der Reifung zellulärer Abwehrmechanismen des Welpen eine wesentliche Rolle [6]. Echinacea und Coffea forcieren die Entwicklung immunologischer Kompetenzen. Dies wurde für Nutztiere belegt [10] und bestätigt auch die Erfahrung in der Kleintierpraxis. Ein bewährtes Immunstimulans bei Kreislaufbeteiligung ist Coffea praeparata oral.

\section{Arzneipflanzen bei Verdauungsstörungen}

Unmittelbar nach der Geburt kann ausschliesslich Milch verdaut werden. Saccharase und Amylase fehlen noch. Der Magen-pH liegt noch hoch, was die Passage von Mikroorganismen in den Dünndarm begünstigt. Dies ist einerseits lebensnotwendig, um die Besiedelung mit Kommensalen zu ermöglichen, kann bei starker bakterieller Kontamination jedoch leicht zu Darminfektionen führen. Mit zunehmendem Alter ist die Säurebarriere des Magens schwieriger zu überwinden. Nur eine welpengerechte Nahrung sowie Rhythmik der Nahrungsaufnahme gewährleisten eine komplikationslose Verdauung [6].

Diarrhöen sind beim Welpen nicht selten. Bei starker Diarrhö droht Säuglingen bald der hypovolämische Kreislaufzusammenbruch. Austrocknungserscheinungen von Haut und Schleimhaut, eingeschränkte Speichelsekretion, Kälte der Extremitäten, beschleunigter, kleiner, weicher Puls und hohe Atemfrequenz weisen auf eine lebensbedrohliche Exsikkose hin und bedürfen der intensivmedizinischen Betreuung. Begleitend sowie bei milden Verläufen und in frühen Stadien einer Diarrhö sind Adsorbenzien wie Kaffeekohle, Heilerde, medizinische Kohle und Tonminerale zu empfehlen, die Bakterientoxine und Gärungsprodukte binden. Pektine sorgen für eine Eindickung des Darminhalts, adsorbieren Toxine und verhindern das Anhaften von Bakterien an die Darmwand. In der Human- und Tiermedizin bewährt ist die Moro'sche Karottensuppe nach einer Rezeptur des Kinderarztes Prof. Dr. Ernst Moro: 500 g geschälte Karotten werden in 11 Wasser für mindestens 1 h gekocht. Anschliessend wird die eingekochte Menge wieder mit heissem Wasser auf 11 aufgefüllt, mit 1 gestrichenen Teelöffel Salz versetzt und püriert. Beim Kochen freigesetzte Oligogalakturonsäuren verhindern das Andocken pathogener Keime an die Darmwand.

Bei Diarrhö haben sich zudem Gerbstoffdrogen bewährt. Diese wirken adstringierend, sekretions- und keimhemmend, dichten durch Vernetzen von Eiweissmolekülen die Darmschleimhaut ab, erschweren dadurch das Eindringen von Toxinen und pathogenen Erregern und hemmen proinflammatorische Enzyme sowie die Darmmotilität. Bei Welpen kommen Drogen mit kondensierten Gerbstoffen (Catechingerbstoffe) wie Blutwurz (Dekokt), Grüntee und Schwarztee zum Einsatz. Beim Hundewelpen bewähren sich auch Teezubereitungen aus getrockneten Heidelbeeren. Hydrolysierbare Gerbstoffe (vor allem Gallotannine) sind bei Katzen grundsätzlich kontraindiziert. Bei der Hydrolyse entstehen insbesondere für Katzen toxische Phenole. Diese können resorbiert werden, was langfristig auch bei anderen Tieren zur Leberschädigung führen kann. Es ist zu beachten, 
dass alle Gerbstoffe dosisabhängig die Schleimhäute reizen und die Resorption von Nahrungsproteinen verzögern. Eine Langzeitanwendung in hoher Dosierung führt $\mathrm{zu}$ Wachstumsstörungen.

Ein bewährtes Multitalent für Mutter und Kind, das auch die Katze verträgt, ist die Kamille. Kamillentee wirkt bei Magen-Darm-Störungen antiphlogistisch, spasmolytisch, antidyspeptisch, cholagog und leicht antimikrobiell.

Ein Problem mutterlos aufgezogener Welpen sind Darmträgheit und Obstipation bzw. Koprostase. Hier fehlt das für Harn- und Kotabsatz nötige Belecken durch die Mutter. Ersatzweise müssen diese Welpen nach den Mahlzeiten mit einem feucht-warmen Tuch in der Anal- und Abdominalregion massiert werden. Auch die Massage der Magenregion ist wichtig, damit verschluckte Luft aufgestossen werden kann. Die Massage kann erst entfallen, wenn die Welpen mobil werden $[6,8]$.

Auch die Umstellung auf feste Nahrung kann zu Darmträgheit und Obstipation, aber auch zu Flatulenz und Meteorismus führen, die für den Welpen oft mit Schmerzen und Krämpfen verbunden sind.

Mittel der Wahl beim Hundewelpen sind milde Bitterstoffdrogen wie die Schafgarbe sowie Karminativa wie Kümmel und Fenchel. Diese wirken sekretions- und appetitanregend, antibakteriell und spasmolytisch. Ätherisches Kümmelöl (0,5-2,0 $\mathrm{ml}$ in $100 \mathrm{ml}$ Olivenöl) eignet sich bei Hundewelpen auch zur Bauchmassage. Es werden 1-2 Tropfen der Mischung mit im Uhrzeigersinn kreisenden Bewegungen einmassiert.

Bei Katzenwelpen wird der Obstipation durch Sahne oder fette pflanzliche Öle entgegengewirkt. Sollen bei ihr Bitterstoffdrogen gegen Flatulenz und Meteorismus eingesetzt werden, kann auf homöopathische Tiefpotenzen zurückgegriffen werden.
Bei Atemwegserkrankungen bewährt sich die Inhalation ätherischer Öle von Kamille und Thymian Ct. Linalool. Einsetzbar sind auch für menschliche Neugeborene empfohlene Ätherisch-Öl-Mischungen aus Lavendel, Melisse, Thymian Ct. Linalool usw., welche die Atemwege befreien und beruhigend, tonisierend, entzündungshemmend und keimreduzierend wirken [11]. Bei Katzenwelpen sind sie geeignet zur Inhalation über die Duftlampe, bei Hundewelpen auch zur Herstellung einer Einreibe-Mischung mit fettem Öl. Als verträglich bei Neugeborenen und Säuglingen gelten z.B. kaltgepresste Öle von Olive, Haselnuss, Mandel, Walnuss, Jojobanuss, Weizenkeimen und Nachtkerzensamen.

Vorsicht geboten ist bei der Anwendung von 1,8-Cineolhaltigen (Eukalyptus, Myrte, Cajeput, Niauli) und Mentholhaltigen (Pfefferminze, Krauseminze) Phytotherapeutika sowie Campher. Hier sind bei menschlichen Säuglingen wegen des starken Geruchs Laryngospasmen, Glottisödeme und reflektorischer Atemstillstand (Kratschmer-HolmgrenReflex) beschrieben worden. Eine Anwendung im Gesicht und grossflächig auf der Haut sollte auch bei Hunde- und Katzenwelpen unterbleiben.

\section{Fazit}

Die Phytotherapie hat zur Gesunderhaltung von Mutter und Kind einiges zu bieten. Doch auch bei der Anwendung von Pflanzenzubereitungen im peripartalen Zeitraum ist besondere Vorsicht geboten, dies insbesondere bei der aus Sicht der Phytotherapie grundsätzlich heiklen Katze.

\section{Disclosure Statement}

Keine Interessenkonflikte.

\section{Literatur}

1 Kumar V: Propolis in dentistry and oral cancer management. N Am J Med Sci 2014;6:250-259.

2 Warad SB, Kolar SS, Kalburgi V, Kalburgi NB: Lemongrass essential oil gel as a local drug delivery agent for the treatment of periodontitis. Anc Sci Life 2013;32:205-211.

3 Wabner D, Beier C (Hrsg): Aromatherapie. München, Elsevier, 2009.

4 Carson CF, Hammer KA, Riley TV: Melaleuca alternifolia (Tea Tree) oil: a review of antimicrobial and other medicinal properties. Clin Microbiol Rev 2006;19:50-62.
5 Löscher W, Richter A, Potschka H: Pharmakotherapie bei Haus und Nutztieren, ed 9. Stuttgart, Enke, 2014.

6 Walser K, Bostedt H (Hrsg): Neugeborenen- und Säuglingskunde der Tiere. Stuttgart, Enke, 1990.

7 Brendieck-Worm C: Aromatherapie; in Bolbecher G, Zurr D (Hrsg): Ganzheitliche Verhaltenstherapie bei Hund und Katze, ed 2. Stuttgart, Sonntag, 2015.

8 Dillitzer N: Tierärztliche Ernährungsberatung, ed 2. München, Urban \& Fischer, 2012.
9 Pentagos Tabares F, Bedoya Jaramillo JV, RuizCortes ZT: Pharmacological overview of galactogogues. Vet Med Int 2014;2014:602894.

10 Kühn T: Paramunisierung neugeborener Kälber mit einem Echinacea-/Coffea-haltigen Präparat. Diss. med. vet. Leipzig, 1994.

11 Stadelmann I: Bewährte Aromamischungen, ed 5. Wiggensbach, Stadelmann, 2007. 